\title{
Absolute photonic band gaps in 12-fold symmetric photonic quasicrystals
}

\author{
Xiangdong Zhang, Zhao-Qing Zhang, and C. T. Chan \\ Department of Physics, The Hong Kong University of Science and Technology, Clear Water Bay, Kowloon, Hong Kong, China
}

(Received 27 October 2000; published 5 February 2001)

\begin{abstract}
A recent publication [Nature (London) 404, 740 (2000)] claimed that absolute photonic gaps can be realized in 12-fold quasicrystalline arrangement of small airholes in a matrix of silicon nitride or glass. The result is rather surprising since silicon nitride $(n=2.02)$ and in particular, glass $(n=1.45)$ have rather low refractive index. In this work, we have studied the transmission properties of the same systems by using the multiplescattering method. We found that the 12-fold triangle-square tiling is indeed very good for the realization of photonic gaps and we found absolute gaps in systems with airholes in dielectric, dielectric cylinders in air, and metal cylinders in air. However, for the case of air-holes in a dielectric background, absolute gaps appear only when the dielectric contrast is sufficiently high, and both silicon nitride and glass have refractive indices below the threshold.
\end{abstract}

DOI: 10.1103/PhysRevB.63.081105

PACS number(s): 42.70.Qs, 41.20.Jb, 74.80.-g

In the past few years there has been a great deal of interest in studying photonic band gap (PBG) materials both theoretically and experimentally. ${ }^{1-9}$ Since a PBG material can have a spectral gap in which electromagnetic wave propagation is forbidden in all directions, it offers the possibility of controlling the flow of photons in a way analogous to electrons in a semiconductor. Furthermore, PBG materials can suppress vacuum fluctuation and spontaneous emission, and can lead to interesting quantum electrodynamics effects. ${ }^{1-3}$ It has potential applications in quantum electronic devices, distributed-feedback mirror, microwave antennae substrate, and its unusual optical properties can be exploited to control and guide the propagation of light. ${ }^{7,8}$ In fact, the prediction that photonic crystals would lead to efficient semiconducting lasers has also been a strong motivation for their development.

Since all the novel properties as well as the application of photonic crystals rely on the existence of $\mathrm{PBG}$, it is essential to identify structures that can support large and robust photonic gaps. So far, many works have been devoted to the study the periodic PBG materials in $2 \mathrm{D}$ and $3 \mathrm{D}$ systems. ${ }^{1-8}$ Large gaps have been obtained in some dielectric or metal odielectric systems. ${ }^{9,10}$ Methods to engineer PBG have also been proposed. ${ }^{11}$ The PBG in periodic systems has been much studied and well understood.

In fact, the PBG exists not only in periodic structures, but also in nonperiodic structures such as quasicrystalline arrangement of dielectrics. Chan et al. ${ }^{12}$ studied and discussed theoretically the PBG in 2D octagonal aperiodic photonic quasicrystals. They found the existence of spectral gaps in these quasiperiodically arranged dielectric system. However, they found that in the octagonal system, the TE and TM spectral gaps usually do not overlap for a reasonable value of the dielectric constant. Recently, Zoorob et al. ${ }^{13}$ claimed that they found absolute photonic gaps in 12-fold symmetric quasicrystals that were realized with small airholes in a silicon nitride or glass waveguide substrate. This is a rather surprising result since both silicon nitride and glass have fairly low refractive indices. If the claims are rigorously correct, the result will have important consequences. Not only that glass is a very common material and is transparent in optical fre- quencies, the fact that the refractive index of glass is only about 1.45 implies that absolute photonic gaps can be achieved with many available materials. In addition, several features reported are also fairly intriguing. For example, the midgap frequency is only very weakly dependent on the filling ratio [Fig. 2(a) of Ref. 13] and silicon-nitride and glass have the first gap at nearly the same frequency [Figs. 2(b) and 2(c) of Ref. 13]. In this work, we have calculated the transmission properties of these 12-fold square-triangle systems using a well-converged multiple-scattering method in $2 \mathrm{D}$. We found that $2 \mathrm{D}$ absolute photonic gaps can indeed appear in 12-fold symmetric quasiperiodic tiling systems if the dielectric contrast is sufficiently high. However, we do not find bona-fide absolute gaps if either silicon nitride or glass is used as the dielectric medium because their refractive indices are too low. If the refractive index contrast is sufficiently large, we show explicitly the existence of absolute gaps for systems with airholes in dielectric and dielectric cylinders in air. Absolute gaps can also easily be realized in "ideal" metal cylinders in air.

In this communication, we will study the gap structures in quasicrystals using the multiple scattering formalism. ${ }^{14} \mathrm{We}$ consider a circular PBG sample of radius $R_{s}$. Inside this circular domain, cylinders of radii $R$ and dielectric constant $\epsilon$ can be arranged in any manner such as a square lattice, triangular lattice, or quasiperiodic lattice. The system is excited by a line source at a fixed frequency located near the center of the sample. The far-field radiated energy flux at the angle $\theta, s(\theta)$, can be calculated by the multiple scattering method, ${ }^{14}$ from which the total radiation power $P_{s}$ $=\int_{0}^{2 \pi} s(\theta) d \theta$ can be obtained. Since the spectrum of $P_{s}$ includes the transmission from all directions, it can be used to determine the position of a complete photonic gap. For frequencies inside a full gap, the density of state is zero, which in turn give a divergent input impedance and vanishing $P_{s}$. Therefore, a gap in the spectrum of $P_{s}$ represents the existence of a complete photonic gap in the system. In order to test the validity of this method, we first calculate $P_{s}$ for the case of square lattice and compare the result with that of band-structure calculations for $S$-polarized ( $E$-field parallel to the cylinders) waves. Figures 1(a) and 1(b) display, re- 


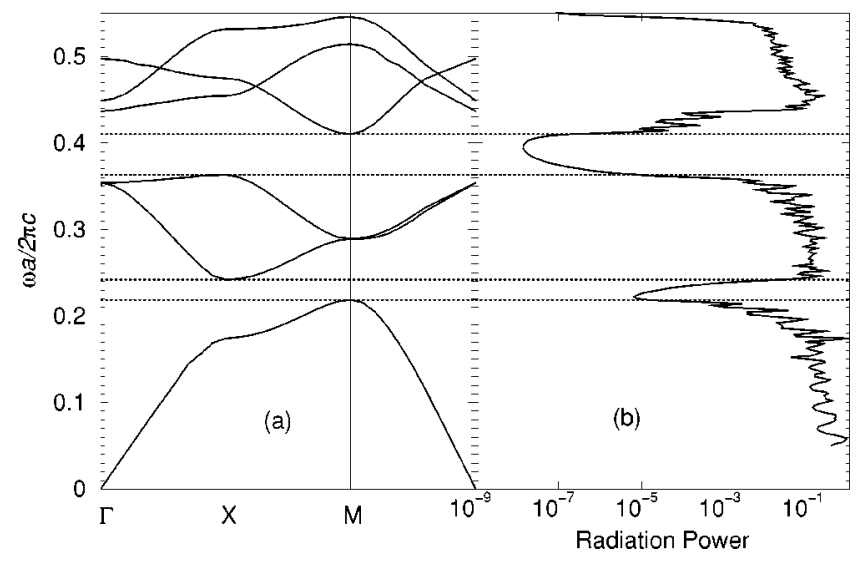

FIG. 1. (a) Calculated photonic band structure of $S$ wave for a square lattice of dielectric cylinder with $R / a=0.38$ and $\epsilon=11.4$. (b) Radiation power for the system being corresponded to (a). The circular PBG sample radius $R_{s}=8$. Dotted lines mark the position of the gap.

spectively, the band structures and radiation power for the case of $\epsilon=11.4$ and $R / a=0.38$, where $a$ is the lattice constant of the square array. The dotted lines mark the position of gaps. The excellent agreement between the results of (a) and (b) in the gap position shows the validity of using the spectrum of radiation power to determine the full gap of a system.

Using the above method, we have calculated the radiation power of the square-triangle tiling system studied in Ref. 13, i.e., a 12-fold symmetric quasicrystalline array of small airholes in silicon nitride $(n=2.02)$. The results for a circular PBG sample of radius $R_{s}=12 a$ are plotted in Fig. 2. Here a is the distance between two cylinders. ${ }^{13}$ The solid line and the dotted line are the results of $P_{s}$ for $S$ and $P$ waves, respectively. For $P$ waves, there is a small gap around the frequency $\omega a / 2 \pi c=0.35$. For $S$ waves, there is no distinct gap between the frequency $\omega a / 2 \pi c=0.15$ and $\omega a / 2 \pi c$ $=0.6$. We thus conclude that there is no absolute gap in the frequency range studied, contrary to the claims of Ref. 13.

In order to further clarify the issue, we have also calculated the transmission coefficient by using the multiple-

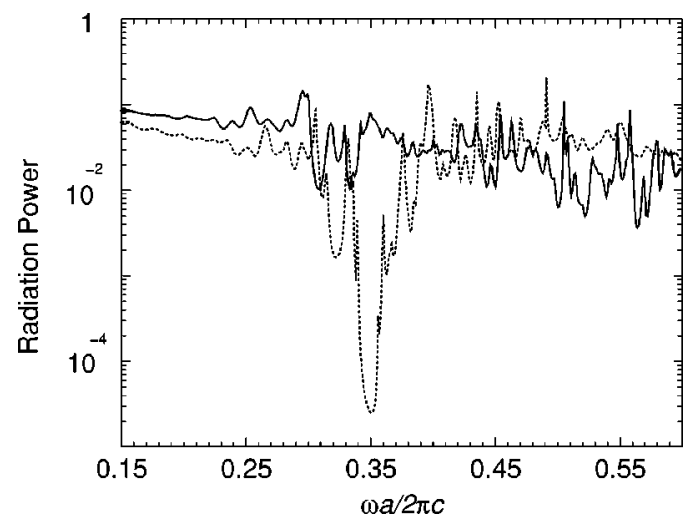

FIG. 2. Radiation power for 12-fold quasicrystal being realized by small airholes in silicon nitride $(n=2.02)$. The structure and parameter are the same as Ref. 13 (air-filling fraction $\beta=28 \%$ ). Solid line is $S$ wave and dotted line $P$ wave.
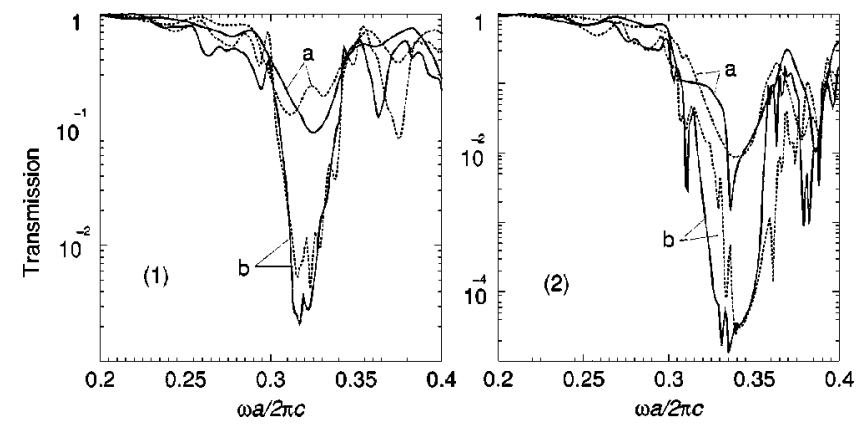

FIG. 3. Transmission coefficient for the 12-fold quasicrystal with the sample width of 31 layers. (1) and (2) describe $S$ and $P$ wave, respectively. (a) 9-layer thickness; (b) 17-layer thickness. The other parameters are the same as Fig. 2. Solid lines correspond to the $\Gamma-X$ direction; dotted lines to the $\Gamma-J$ direction.

scattering method. This method is capable of reproducing accurately the experimental transmission data. ${ }^{11,14}$ The samples considered here are 31 layers in width. The incident beam is prepared by passing a plane wave at a fixed frequency through an open slit in front of the sample. The width of the slit is chosen as $20 \mathrm{a}$, which is smaller than the sample width to avoid diffraction.

For $S$ waves, the calculated transmission coefficients for two samples with thicknesses of 9 and 17 layers are plotted in Fig. 3(1). The solid and dotted lines are the results for the $\Gamma-X$ and $\Gamma-J$ directions, respectively, whereas (a) and (b) denote the results from 9-layer and 17-layer samples, respectively. The corresponding results for $P$ waves are shown in Fig. 3(2). First of all, we note that the transmission spectra is rather isotropic, in the sense that the transmission along $\Gamma$ $-X$ and $\Gamma-J$ are rather similar in their salient features. This is a special property of quasicrystalline geometry. For $P$ waves, the transmission has a dip around $\omega a / 2 \pi c=0.35$ for both directions. Furthermore, the dips increase in depth with the sample thickness. This clearly indicates the existence of a full gap, in agreement with the result of radiation power shown in Fig. 2. However, for $S$ waves the situation is different. We first note the very different scale between the left and the right panel in Fig. 3. The dip for the $S$ waves is much shallower. Although the transmission spectrum has a dip around $\omega a / 2 \pi c=0.32$ for both directions, some peaks are identified inside the dip in the $\Gamma-J$ direction. The number of peaks increase with the sample size. This indicates that there are actually states inside the dip of the spectra, and thus the dip around $\omega a / 2 \pi c=0.32$ is not a spectral gap. In order to understand the property of these states, we have calculated the intensity distribution of the scattered field at the frequency corresponding to the peak position $\omega a / 2 \pi c=0.321$ of the $S$-wave transmission in the $\Gamma-J$ direction for the 9-layer sample. The result in an area of $10 \mathrm{a} \times 10 \mathrm{a}$ space is plotted in the lower panel of Fig. 4(a). Here, the wave enters the surface at $Y=1$ and exits at $Y=9$. The $X$ coordinate shown in the figure covers the central part of the sample. Although the overall intensity decays in the $Y$ direction, there exists a number of local peaks near the center of the sample. The presence of these spatial peaks implies the existence of a localized state inside the dip. The eigenmodes in this spectral 


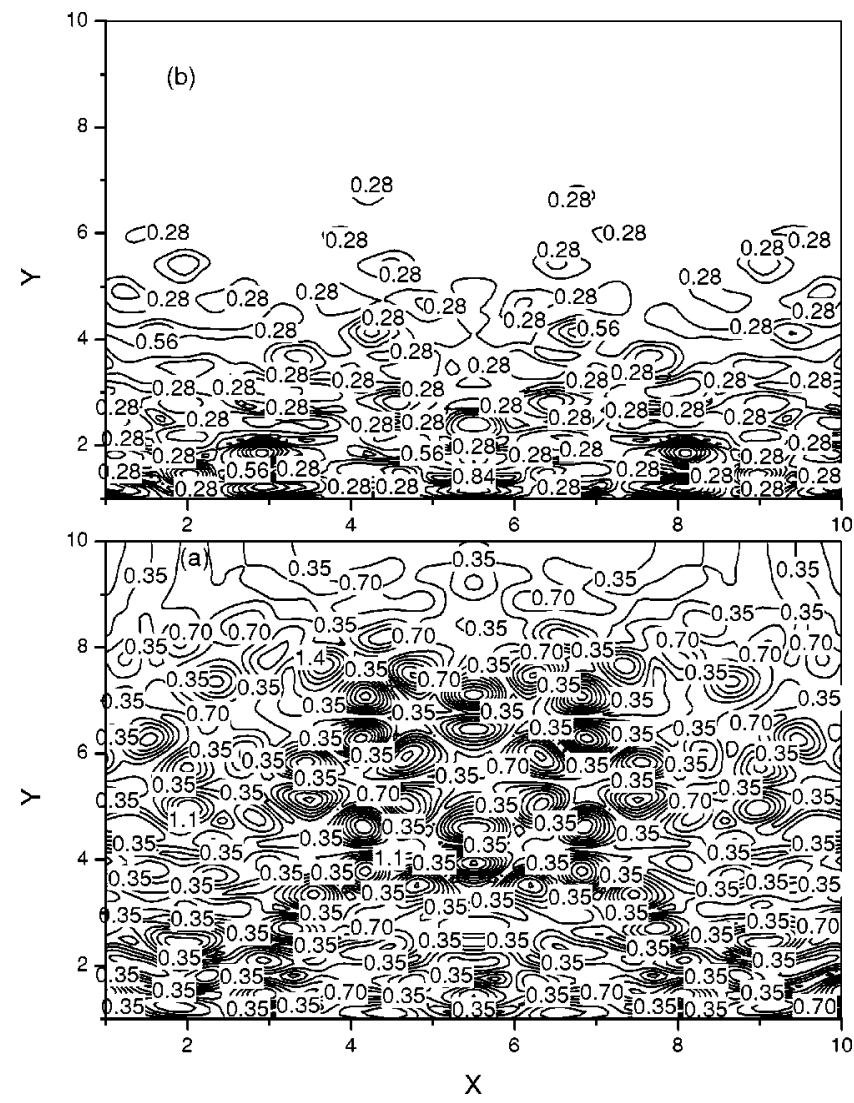

FIG. 4. The field energy distribution for $S$ wave at $\omega a / 2 \pi c$ $=0.321$ (a) and $P$ wave at $\omega a / 2 \pi c=0.35$ (b) in the $\Gamma-J$ direction. The structure and parameter are the same as Fig. 3.

region are highly localized and couples poorly to incident waves, leading to poor transmittance. In the absence of these localized states, the intensity would have decayed exponentially along the $Y$ direction. To demonstrate this, we also plot in the upper panel of Fig. 4(b) the intensity distribution of the scattered field at frequency $\omega a / 2 \pi c=0.35$ inside the $P$-wave gap in the $\Gamma-J$ direction. Clearly there exist no local peaks and, therefore, no signatures of localized states in this case. The dip near $\omega a / 2 \pi c=0.35$ for the $P$ wave is a true spectral gap.

Combining the results of radiation power, transmission spectra, and field energy distributions, we can safely conclude that there is no spectral gap for $S$ waves in the frequency region we studied here. However, for $P$ waves, we find a small gap around $\omega a / 2 \pi c=0.35$. Thus, such a system does not support absolute gaps. The results of Fig. 3 shown here are thus different from the results of theoretical calculation presented in Fig. 2 of Ref. 13. We have also investigated the airhole-in-glass system reported in Ref. 13. Again, we do not find any absolute gaps in such system, which is not surprising since the refractive index of glass is even lower.

Although we do not find absolute gaps in the systems reported in Ref. 13, the 12-fold quasiperiodic square-triangle structure does have the advantage of supporting absolute gaps because of its high symmetry. The only problem with silicon nitride and glass is that the refractive index is not

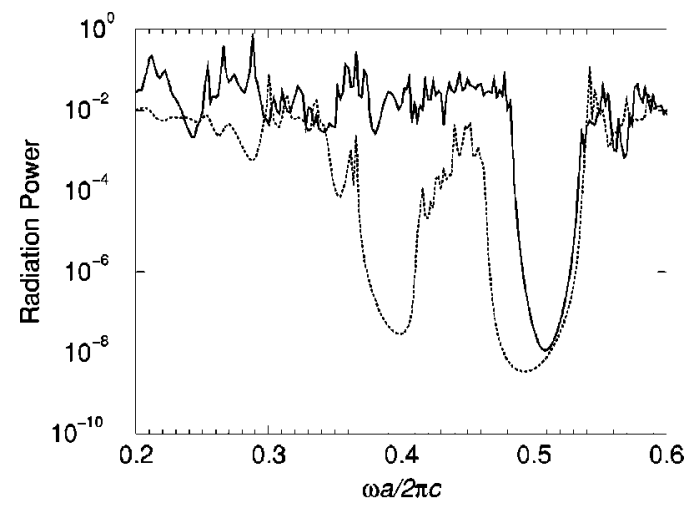

FIG. 5. Radiation power for 12-fold quasicrystal with aircylinder radius $R / a=0.47$. The dielectric constant of background is 8.9. The circular PBG sample radius $R_{s}=10$. Solid line is $S$ wave; dotted line is $P$ wave.

high enough. In periodic structures, light experiences different effective periods as it travels through the crystals along different directions. The existence of a full gap requires the overlap of all directional gaps. Quasicrystalline structure is more isotropic and the square-triangle structure should be very favorable. For this reason, we have done a careful search for the existence of absolute gaps by increasing the dielectric contrast. In the case of airholes in dielectric discussed before, we find that an absolute gap appears when the background dielectric constant is above $\epsilon=7.0$ and the radius of airhole is $R / a=0.47$. For instance, in Fig. 5 we show the radiation power of both $S$ waves (solid line) and $P$ waves (dotted line) for the case of $\epsilon=8.9$ and $R / a=0.47$. For $S$ waves there is a spectral gap around $\omega a / 2 \pi c=0.5$ and this gap overlaps with the second gap of $P$ waves to form an absolute gap.

In fact, the absolute gap does not only exist in the case of airholes in dielectric, it also exists in the case of dielectric cylinders in air. For instance, when the dielectric constant of the cylinders is $\epsilon=11.4$, absolute gaps appear for $R / a$ in the range $(0.35,0.43)$. When $R / a=0.4$, the results of radiation power for $S$ and $P$ waves are shown in Fig. 6 in the solid and dotted lines, respectively. A small overlap around $\omega a / 2 \pi c$ $=0.55$ forms an absolute gap in such system.

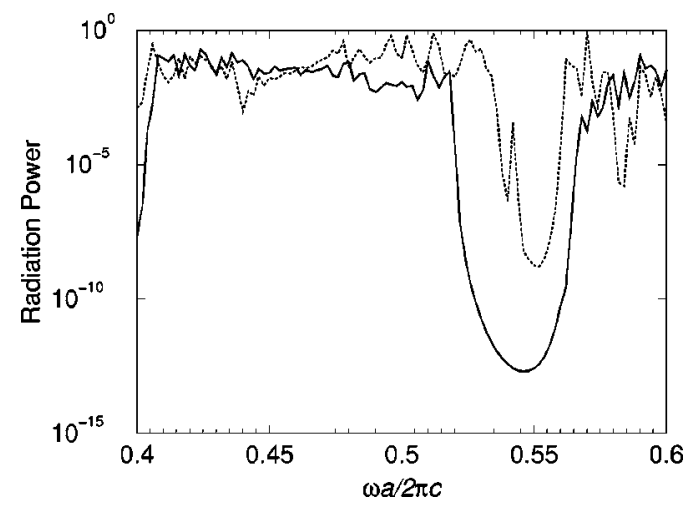

FIG. 6. Radiation power for 12-fold quasicrystal with dielectric cylinder radius $R / a=0.4$ and $\epsilon=11.4$. The circular PBG sample radius $R_{s}=12$. The solid line is $S$ wave; dotted line is $P$ wave. 


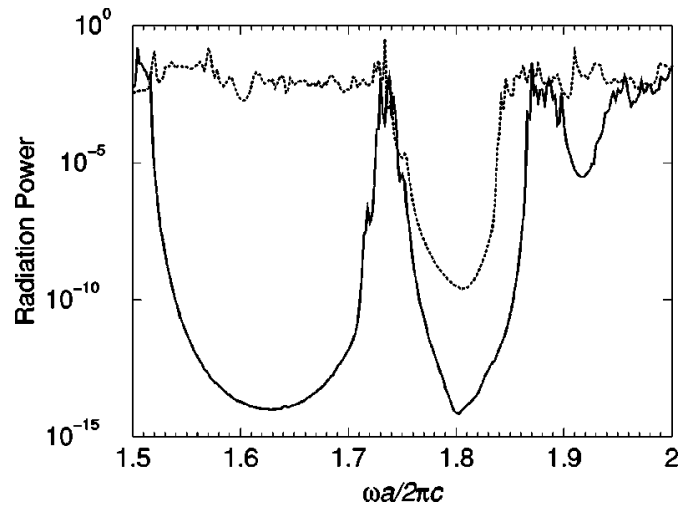

FIG. 7. Radiation power for 12-fold quasicrystal which consist of perfect metal cylinder with $R / a=0.365$ and $\epsilon=-1000$. The circular PBG sample radius $R_{s}=8$. Solid line is $S$ wave; dotted line is $P$ wave.

Finally, we consider the case of metallic cylinder in air. For metallodielectric PBG materials, it has been shown that when the frequency is much smaller than the plasma frequency, the absorption is small and the PBG structures is close to that of perfect metal cylinders. ${ }^{15-17}$ In our calculations, we take the dielectric constant of the "perfect" metal as $\epsilon=-1000$, which serves to reject fields from the metallic domain. The radiation power for the case of $R / a=0.365$ is shown in Fig. 7 for both $S$ waves (solid line) and $P$ waves (dotted line). A large absolute gap exists around $\omega a / 2 \pi c$ $=1.8$.

In conclusion, we studied the transmission properties of 12-fold square-triangle photonic quasicrystals using the multiple scattering method. We found that such structures indeed support absolute photonic gaps as long as the dielectric contrast is sufficiently high. These quasiperiodic systems are actually quite potent in supporting photonic gaps since it is rather rare for the same structure to support absolute gaps in the air-in-dielectric as well as the inverted dielectric-in-air configuration. However, we do not find absolute gaps for the air-in-dielectric configuration if low refractive material such as silicon-nitride and glass are used as the dielectric medium. Although in the 2D multiple-scattering calculations presented here the third dimension is assumed to be infinite in length, nevertheless, our main conclusions can apply to real samples with a finite length in the third dimension. In fact, it has been shown that the method used here is capable of reproducing accurately the measured transmission data in two different experiments. ${ }^{11,14,18}$

This work was supported by Hong Kong RGC Grant No. HKUST 6160/99P. C.T.C. was supported by HKUST6136/ 97P.
${ }^{1}$ See, for example, Photonic Band Gaps and Localization, edited by C.M. Soukoulis (Plenum, New York, 1993); J. Opt. Soc. Am. B 10, 208-408 (1993); Photonic Band Gap Materials, edited by C.M. Soukoulis (Kluwer, Dordrecht, 1996).

${ }^{2}$ J.D. Joannopolous, R.D. Meade, and J.N. Winn, Photonic Crystals (Princeton University, Princeton, 1995).

${ }^{3}$ E. Yablonovitch, Phys. Rev. Lett. 58, 2059 (1987).

${ }^{4}$ S. John, Phys. Rev. Lett. 58, 2486 (1987).

${ }^{5}$ K.M. Leung and Y.F. Liu, Phys. Rev. Lett. 65, 2646 (1990); Z. Zhang and S. Satpathy, ibid. 65, 2650 (1990); K.M. Ho, C.T. Chan, and C.M. Soukoulis, ibid. 65, 3152 (1990).

${ }^{6}$ S.Y. Lin et al., Nature (London) 394, 251 (1998).

${ }^{7}$ A. Mekis et al., Phys. Rev. Lett. 77, 3787 (1996).

${ }^{8}$ J.C. Knight et al., Science 282, 1476 (1998).

${ }^{9}$ C.M. Anderson and K.P. Giapis, Phys. Rev. Lett. 77, 2949 (1996).
${ }^{10}$ N.A. Nicorovici et al., Phys. Rev. B 52, 1135 (1995).

${ }^{11}$ X. Zhang et al., Phys. Rev. B 61, 1892 (2000); S. J. Cox and D. C. Dobson, SIAM (Soc. Ind. Appl. Math.) J. Appl. Math. 59, 2108 (1999).

${ }^{12}$ Y.S. Chan, C.T. Chan, and Z.Y. Liu, Phys. Rev. Lett. 80, 956 (1998); S.S. Cheng, L.M. Li, C.T. Chan, and Z.Q. Zhang, Phys. Rev. B 59, 4091 (1999).

${ }^{13}$ M.E. Zoorob et al., Nature (London) 404, 740 (2000).

${ }^{14}$ L.M. Li and Z.Q. Zhang, Phys. Rev. B 58, 9587 (1998); G. Tayeb and D Maystre, J. Opt. Soc. Am. A 14, 3323 (1997).

${ }^{15}$ L.M. Li, Z.Q. Zhang, and X. Zhang, Phys. Rev. B 58, 15589 (1998).

${ }^{16}$ M.M. Sigalas, C.T. Chan, K.M. Ho, and C.M. Soukoulis, Phys. Rev. B 52, 11744 (1995).

${ }^{17}$ V. Kuzmiak and A.A. Maradudin, Phys. Rev. B 55, 7427 (1997).

${ }^{18}$ C. Jin et al., Appl. Phys. Lett. 75, 1201 (1999). 